\title{
Prognostic impact of a compartment-specific angiogenic marker profile in patients with pancreatic cancer
}

\author{
Christoph Kahlert' ${ }^{1,}$, Maria Fiala1, ${ }^{1,}$, Gabriel Musso ${ }^{2,3}$, Niels Halama ${ }^{4}$, Sophia Keim4, \\ Massimiliano Mazzone ${ }^{5,6}$, Felix Lasitschka7, Mathieu Pecqueux ${ }^{8}$, Fee Klupp ${ }^{1}$, \\ Thomas Schmidt ${ }^{1}$, Nuh Rahbari $^{8}$, Sebastian Schölch ${ }^{8}$, Christian Pilarsky ${ }^{8}$, \\ Alexis Ulrich ${ }^{1}$, Martin Schneider ${ }^{1}$, Juergen Weitz ${ }^{8}$, Moritz Koch ${ }^{8}$ \\ ${ }^{1}$ Department of General, Visceral and Transplantation Surgery, University of Heidelberg, Heidelberg 69120, Germany \\ 2 Department of Medicine, Harvard Medical School, Boston, MA 02115, USA \\ ${ }^{3}$ Cardiovascular Division, Brigham and Women's Hospital, Boston, MA 02115, USA \\ ${ }^{4}$ Medical Oncology, National Center for Tumor Diseases and Hamamatsu Tissue Imaging and Analysis (TIGA) Center, Institute \\ for Medical Biometry and Informatics, University of Heidelberg, Germany \\ ${ }^{5}$ Laboratory of Molecular Oncology and Angiogenesis, Vesalius Research Center, VIB, Leuven 3000, Belgium \\ ${ }^{6}$ Laboratory of Molecular Oncology and Angiogenesis, Vesalius Research Center, Department of Oncology, KU, Leuven 3000, \\ Belgium \\ ${ }^{7}$ Institute of Pathology, University of Heidelberg, Heidelberg 69120, Germany \\ ${ }^{8}$ Department of General, Visceral and Thoracic Surgery, University of Dresden, Dresden 01307, Germany \\ *These authors contributed equally to this work
}

Correspondence to:

Christoph Kahlert, e-mail: christoph.kahlert.079@googlemail.com

Keywords: Pancreatic cancer, stroma, angiogenic cytokines, expression profile

Received: August 05, 2014

Accepted: October 27, 2014

Published: December 30, 2014

\section{ABSTRACT}

Pancreatic cancer consists of a heterogenous bulk of tumor cells and stroma cells which contribute to tumor progression by releasing angiogenic factors. Those factors can be detected as circulating serum factors. We performed a compartmentspecific analysis of tumor-derived and stroma-derived angiogenic factors to identify biomarkers and molecular targets for the treatment of pancreatic cancer. Kryo-frozen tissue from primary ductal adenocarcinomas $(n=51)$ was lasermicrodissected to isolate tumor and stroma tissue. Expression of 17 angiogenic factors (angiopoietin-2, follistatin, GCSF, HGF, interleukin-8, leptin, PDGF-BB, PECAM-1, VEGF, matrix metalloproteinase -1, -2, -3, -7, -9, -10, -12, and -13) was analyzed using a multiplex elisa assay for tissue-derived proteins and corresponding serum.

Our study reveals a compartment-specific expression profile for several angiogenic factors and matrix metalloproteinases. ROC analysis of corresponding serum samples reveals MMP-7 and MMP-12 as strong classifiers for the diagnosis of patients with pancreatic cancer vs. healthy control donors. High expression of tumor-derived PDGF-BB and MMP-1 correlates with prolonged survival in univariate and multivariate analysis. In conclusion, a distinct expression patterns for angiogenic cytokines and MMPs in pancreatic cancer and surrounding stroma may implicate them as novel targets for cancer treatment. Tumor-derived PDGF-BB and MMP-1 are significant and independent prognostic markers for poor survival. 


\section{INTRODUCTION}

Pancreatic cancer is the fourth leading cause of cancer-related death in the Western hemisphere $[1,2]$. Recent improvements in the treatment of pancreatic cancer by multidisciplinary therapeutic approaches and the introduction of more effective chemotherapy regimens such as the combination of gemcitabine with albuminbound paclitaxel or the combination of oxaliplatin, irinotecan, fluoruracil, and leucovorin (FOLFIRINOX) have increased significantly the prognosis of a selected group of patients [3-5]. However, the cumulative overall 5-year survival of all patients with pancreatic cancer remains less than $5 \%$, with a median survival of 4 to 6 months [6]. Curative resection through surgery is only possible for approximately $15-20 \%$ of cases, and even in these patients, the median survival reaches only 28 months [6].

In an effort to improve the clinical outcome of pancreatic cancer, there is an increasing focus on tailoring therapeutics to individual tumor biology [7]. This involves the use of prognostic biomarkers, including lymph node status, tumor differentiation grade, and tumor size [8-10]. While promising, additional biomarkers are actively sought in an attempt to further stratify patients into different risk groups that might benefit from a more individualized therapy.

In addition to tissue obtained from the primary tumor cells, the adjacent tumor stroma provides an important source of potentially prognostic or predictive biomarkers in tumor disease [11-13]. In these compartments, biomarkers can derive either directly from tumor cells or are indirecte surrogates for the systemic inflammatory response of the host $[14,15]$. For example, fibrotic stroma is a major component of the pancreatic tumor bulk [16], consisting of an extracellular matrix and various cell types such as pancreatic stellate cells, endothelial cells and inflammatory cells [16]. The intercellular cross-talk between cancer cells and the tumor microenvironment via angiogenic cytokines or growth factors is essential for tumor progression and dissemination [17]. Moreover, tumor-stroma-associated cells secrete matrix metalloproteinases which can degrade the extracellular matrix and contribute to tumor angiogenesis $[18,19]$. Therefore, in addition to tumor epithelial-derived biomarkers, tumor stroma-secreted proteins may supply detailed information about the tumor biology and present novel molecular targets for the treatment of pancreatic cancer [20].

To address this, we examined the suitability of tumor-, stroma- and serum-derived proteins as biomarkers in pancreatic cancer. To do so, we separated cancer cells and surrounding tumor stroma, subsequently comparing the expression of 9 angiogenic cytokines and 8 matrixmetalloproteinases (MMPs) by using the new technology of a multiplex-based angiogenic cytokine and MMP assays.
Our study shows a compartment-specific expression profile of angiogenic factors in pancreatic cancer. In corresponding serum samples, we identified serum-derived MMP-7 and MMP-12 as strong classifiers to diagnose patients and tumor-derived PDGF-BB and MMP-1 as potential prognostic biomarkers in pancreatic cancer.

\section{RESULTS}

\section{Patient characteristics and clinical specimens}

51 patients with pancreatic cancer were included in this study and represent a homogenous cohort (Supplementary Table S1). The median age was 67 years, 29 patients were male, 22 female. The UICC stage at time of tumor resection was II in 8 cases, III in 39 cases and IV in 4 cases. Those patients with stage IV pancreatic cancer had either distant lymph node metastases $(n=2)$ or single, small liver metastases $(n=2)$ and had undergone a macroscopically complete tumor resection. Therefore, stage IV patients in our cohort received a similar postoperative treatment and follow-up with a curative attempt as patients with stage II or stage III pancreatic cancer. 28 specimens were diagnosed with a tumor grade of 2, 23 specimens were diagnosed with a tumor grade of 3. Accoring to the definition of the Leeds Pathology Protocol (LEEPP) [21], the tumor was completely resected (resection status R0) in 7 cases, tumor infiltration was proven at the resection margin during pathological analysis of the removed specimen (resection status R1) in 44 cases, none of the patients presented a macroscopic residual disease (resection status R2). One patient did not receive adjuvant treatment as he was not eligible for the inclusion criteria of an adjuvant chemotherapy due to his general state of health (postoperative Karnofsky index $<60 \%$ ); 50 patients received postoperative chemotherapy.

\section{Expression profile of MMPs and angiogenic cytokines in serum, cancer and stromal cells}

Tumor and corresponding stroma and serum samples were examined from each patient in a standardized manner (see Methods). First, we analyzed the expression of 9 angiogenic cytokines and 8 MMPs in tumor cells, tumor stroma and serum of the same patient (Figure 1, Supplementary Table S2). In 1 of the 51 tissue samples and 3 of 51 serum samples, the multiplex measurement failed and data were excluded from further analysis. Additionally, MMP-3, MMP-12, and MMP-13 could only be detected in fewer than $25 \%$ of the tissue samples, and thus these MMPs were excluded from further correlation analysis. Analysis of angiogenic markers from all three compartments (tumor cells, stroma cells, and serum) revealed high serum expression for five angiogenic cytokines and five MMPs as compared to tumor and stroma tissue 
( $p<0.001$; Figure 1): angiopoietin-2, follistatin (tumor only), CSF, leptin, PDGF-BB, MMP-1,-2,-7,-9 and -10 . Serum-derived PECAM-1 displayed a significantly lower expression in serum, as compared to stroma tissue $(p<0.001)$. Alternately, MMP-7 had increased expression in tumor tissue as compared to stroma $(p<0.001)$. Follistatin was significantly upregulated in tumor stroma, as compared to tumor cells $(p<0.001)$ while G-CSF, HGF and MMP-2 showed a trend for an increased expression in the stromal compartment. Pearson correlation between serumderived factors and tissue-derived factors revealed no significant linear relationship for any angiogenic cytokine nor any MMP (Supplementary Table S3, Figure 1).

\section{Evaluation of serum MMPs as pancreatic cancer diagnostic biomarkers}

The role of angiogenic cytokines as potential serum biomarkers to distingish between patients with pancreatic cancer and healthy donors has already been adressed $[12,22]$. Therefore, we focused the next phase of our analysis on the circulating MMPs as potential diagnostic markers to discriminate between patients with pancreatic cancer and healthy individuals. As control cohort, serum samples of 44 healthy donors were included. MMP-1,-3,-7,-9,-10 and -12 were significantly upregulated in patients with pancreatic cancer
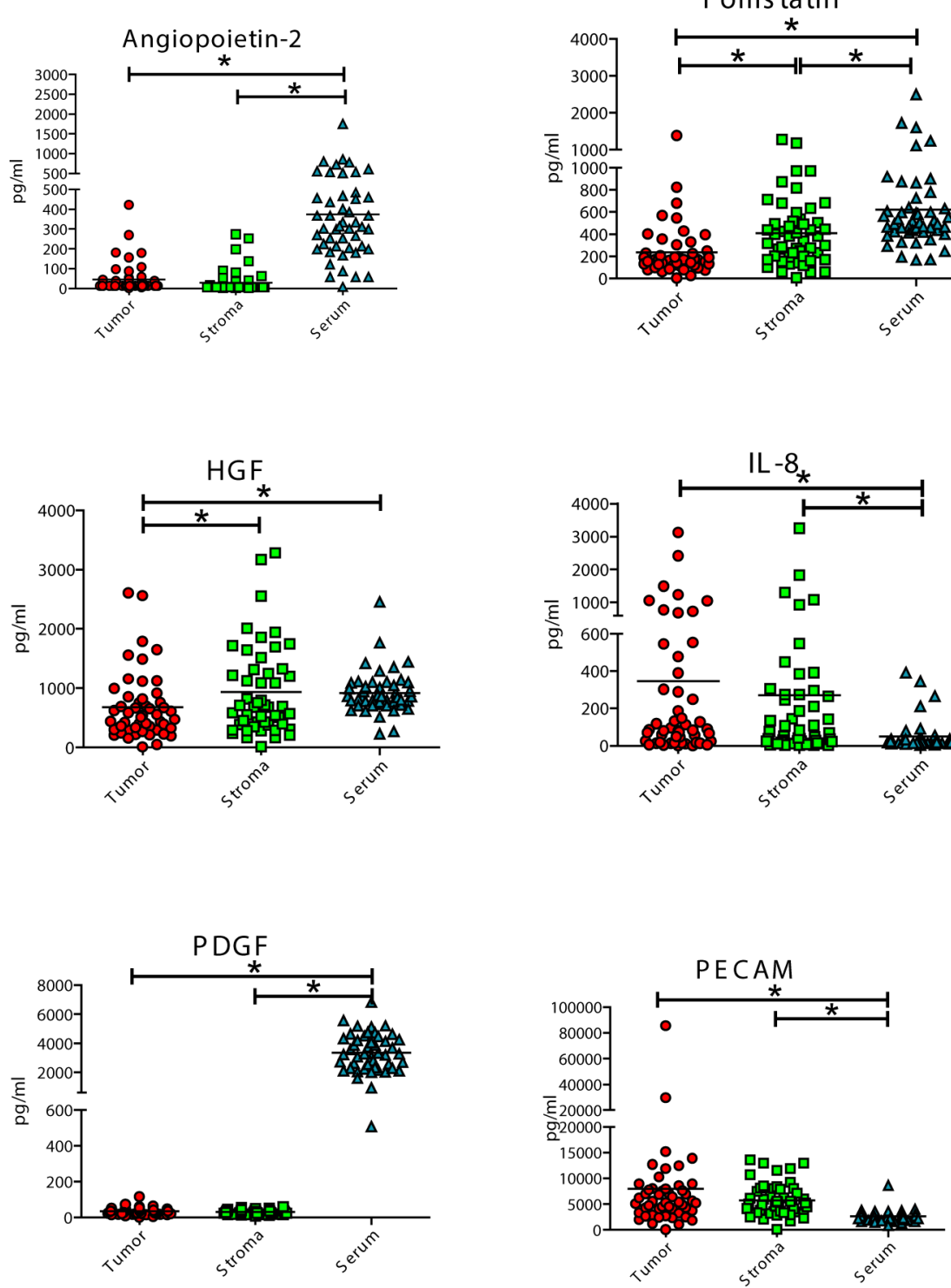
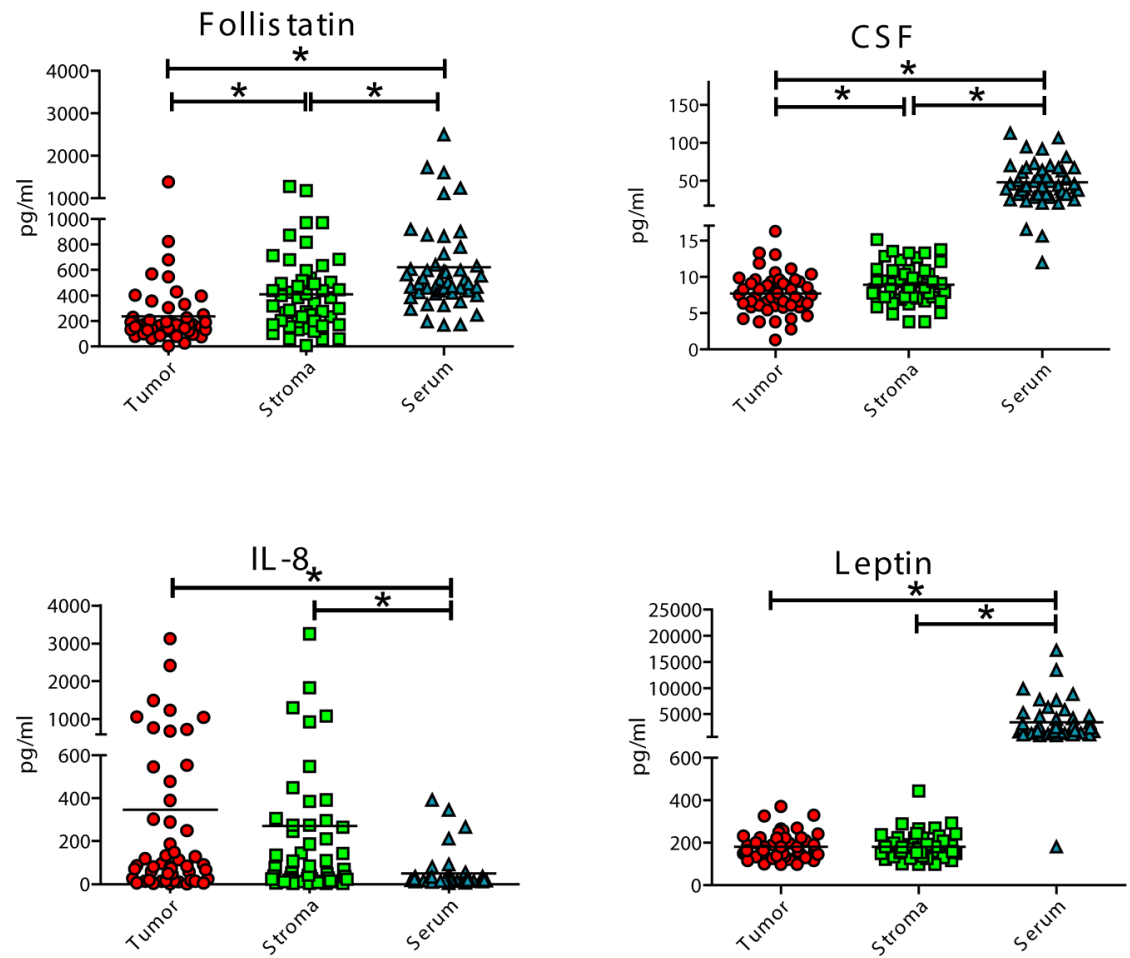

Figure 1: Expression analyis of angiopoietin-2, follistatin, granulocyte colony-stimulating-factor, (G-CSF), hepatocyte growth factor (HGF), interleukin-8 (IL-8, CXCL8), leptin, platelet-dereived growth factor beta (PDGF-BB), platelet endothelial cell adhesion molecule-1 (PECAM-1, sCD31), vascular endothelial growth factor (VEGF).

(Continued) 

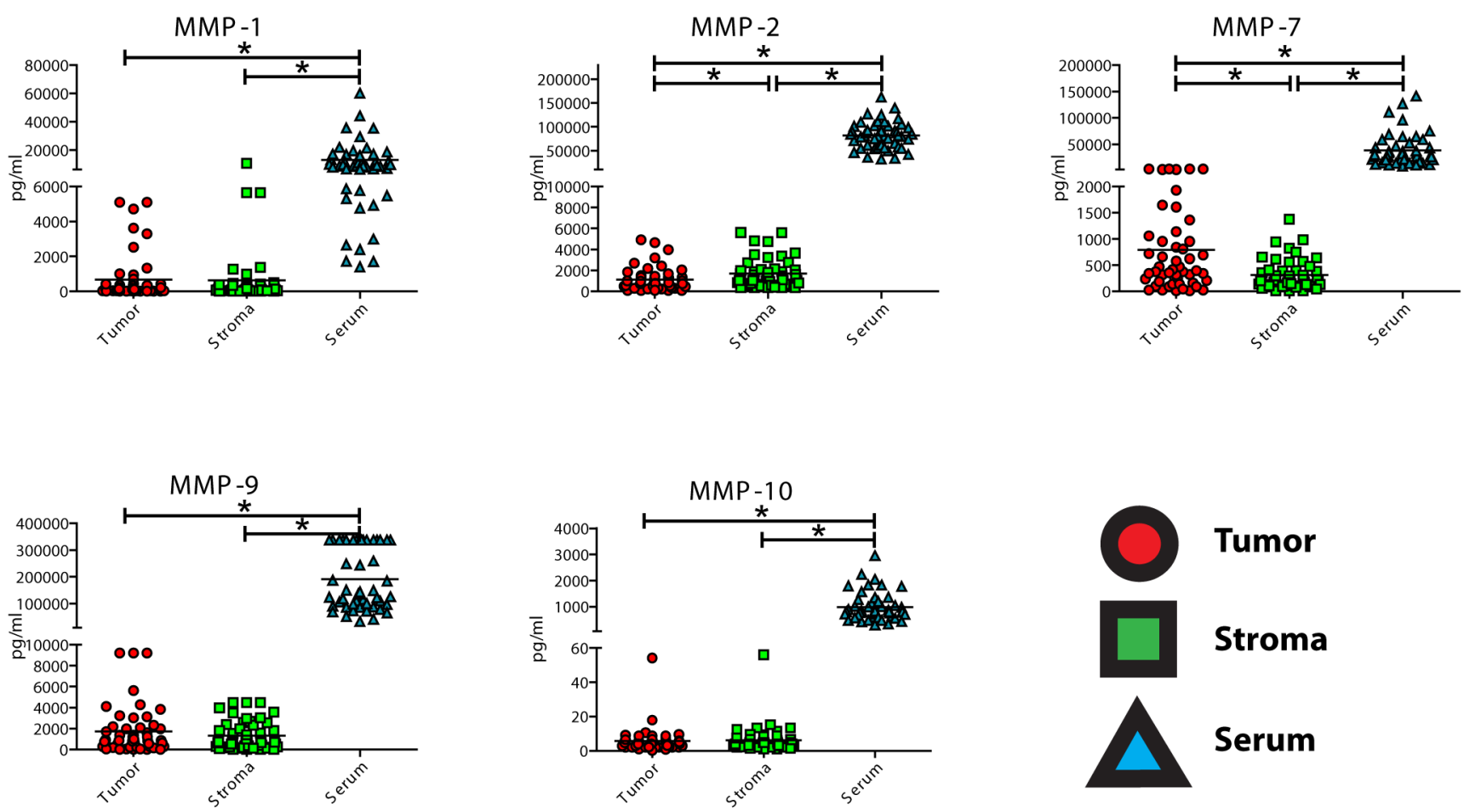

Figure 1 (Continued): Matrix metalloproteinase (MMP)-1, -2, -7, -9, and -10 in tumor epithelial cells, tumor-associated stroma, and corresponding serum samples. The concentratons are given as $\mathrm{pg} / \mathrm{ml}$ for each parameter. $\left(^{*}\right)$ marks a signficant difference $(p<0.05)$ between the expression of angiogenic cytokines or MMPs among the three different compartments.

( $p<0.0001$, respectively), whereas MMP-2 was significantly decreased in the cancer patient group ( $p<0.0001$; Figure 2A). While all MMPs served as good predictors of disease, as assessed using area under the receiver operating characteristic curve (AUC) (see Table 1), MMP-7 and MMP-12 are perfect classifiers (MMP-7: AUC $=0.97$, sensitivity $=90 \%$, specificity $=100 \%$ MMP$12:$ AUC $=1$, sensitivity $=100 \%$, specificity $=100 \%$ ), suggesting them as meaningful diagnostic biomarkers in pancreatic cancer. Subgroup ROC analysis of stage II - IV pancreatic cancer revealed similar results for MMP-7 and MMP-12 (Supplementary Table S4).

\section{Compartment-specific angiogenic marker profile of MMPs and angiogenic cytokines in correlation to clinicopathological parameters and clinical outcome}

To evaluate the prognostic relevance of tumorderived, stroma-derived, and serum-derived angiogenic markers, the expression of each factor was correlated with clinical outcomes and histopathological parameters. The patient cohort was divided into groups based on expression, such that those having expression of angiogenic cytokines or MMPs below the median were considered low, and all else considered high. Chi square tests revealed no statistically significant correlation between any tumor-derived, stroma-derived or serum-derived angiogenic factor (Supplementary Table 5 - 11). Unsupervised hierachical clustering based on expression of angiogenic cytokines and MMPs did not reveal any distinct subsets of patients for tumor-, serum-, or stroma-derived factors (Supplementary figure 1). Similarly, no distinct clusters were apparent when supervising cluster analysis based on patient tumor grade or UICC stage of the primary cancer (data not shown).

Alternately, univariate analysis (see Methods) revealed that the expression of three angiogenic factors was predictive of survival in our patient cohort $(p<0.05$; Figure 3 \& Table 2). Specifically, high expression of tumorderived PDGF-BB and MMP-1 were associated with increased cancer survival $(p<0.05)$, while high expression of MMP-2 showed a trend towards improved outcome $(p=0.058)$ (see Table 2). Notably, survival analysis did not show any significant associations between expression of any stroma- or serum-derived angiogenic cytokine or MMP and clinical outcome. Using multivariate testing (Table 3), which includes all relevant clinicopathological parameters as well as all angiogenic cytokines and MMPs, high expression of tumor-derived PDGF-BB and high expression of tumor-derived MMP-1 were independent prognostic markers for cancer-specific survival (PDGFBB: Odds ratio: 0.265, CI: 0.101-0.693, $p=0.007$; MMP-1: Odds ratio: 0.347, CI: 0.121-0.996, $p=0.049$ ). 

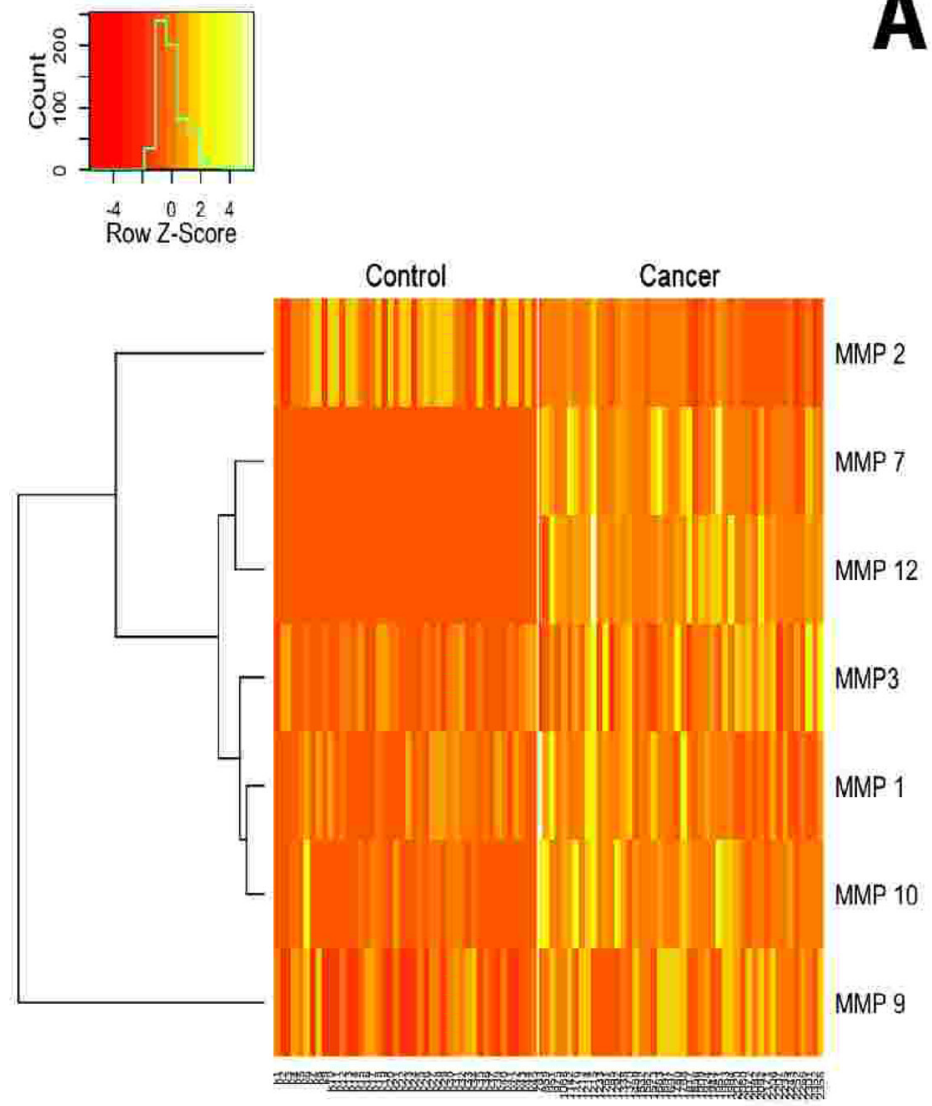

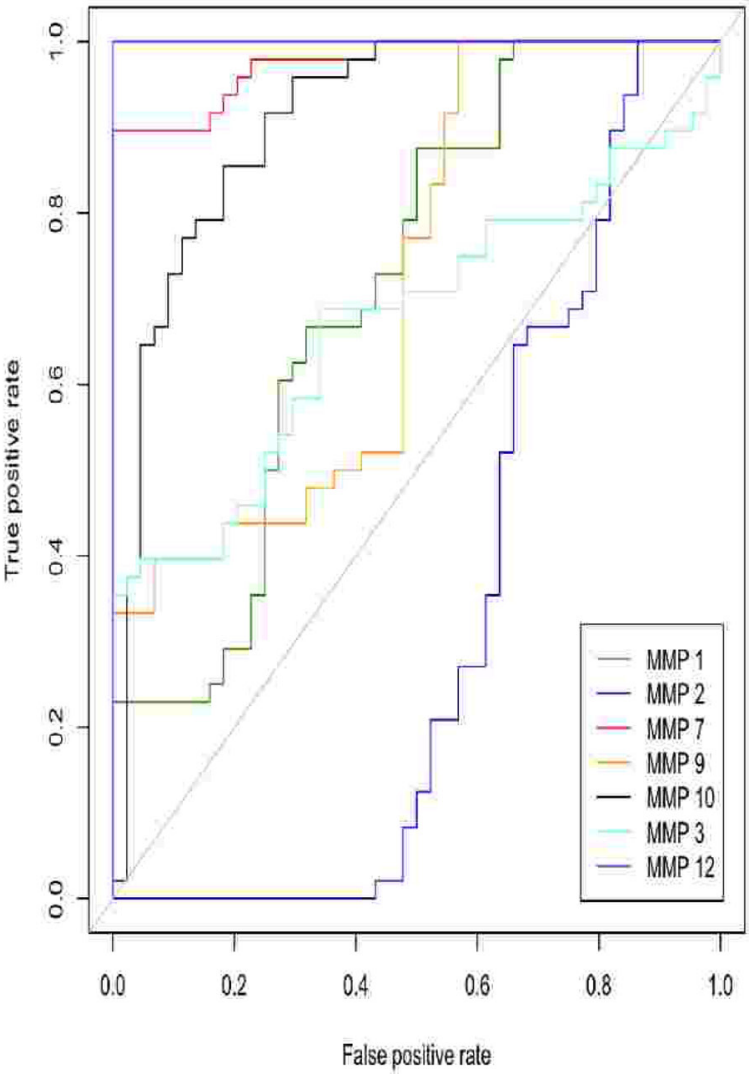

Figure 2: (A) The heatmap displays the expression pattern of serum MMPs in samples from healthy donors (control) and patients with pancreatic cancer. MMP-2 was significantly downregulated in PDAC serum samples compared to control $(p>0.0001)$, whereas MMP-1,-3,-7,-9,-10 and -12 were significantly increased in cancer patients $(p>0.0001)$. (B) Area under the receiver operating characteristic (ROC) curves for all MMPs with samples from patients with pancreatic cancer and the control group.

Table 1: Area under the receiver operating characteristic curves (AUC) of serum MMPs for the diagnosis of patients with pancreatic cancer vs. healthy control donors. The cutoff was determined using the Youden-Index.

\begin{tabular}{|l|llll|l|}
\hline Serum marker & AUC & Cutoff & Sensitivity & \multicolumn{1}{l|}{ Specificity } \\
\hline MMP-1 & 0.71 & 7754.2 & 0.67 & 0.68 \\
\hline MMP-2 & 0.34 & 75210.8 & 0.65 & 0.34 \\
\hline MMP-3 & 0.67 & 14787.72 & 0.69 & 0.66 \\
\hline MMP-7 & 0.97 & 12324.5 & 0.9 & 1 \\
\hline MMP-9 & 0.71 & 90602.1 & 0.77 & 0.52 \\
\hline MMP-10 & 0.91 & 559.5 & 0.85 & 0.82 \\
\hline MMP-12 & 1 & 2215.58 & 1 & 1 \\
\hline
\end{tabular}

\section{DISCUSSION}

Pancreatic cancer is composed of a heterogenous bulk of tumor cells and adjacent stroma cells, with both of these compartments regulating tumor progression and dissemination through the release of angiogenesisassociated cytokines and matrix-metalloproteinases [17].

In this context, the functional role of the tumor stroma remains ambiguous. Some components of the tumor stroma such as activated cancer-associated 

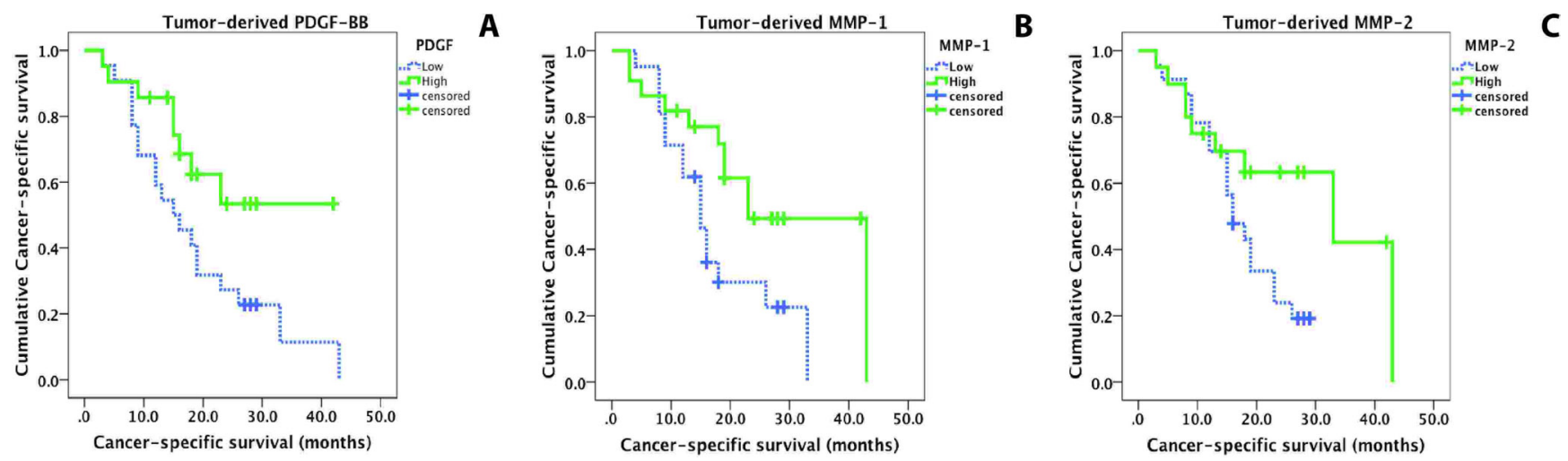

Figure 3: Kaplan-Meier curves display cancer-specific survival in patients with pancreatic cancer. Solid line: Low expression; dashed line: high expression. (A) Overexpression of tumor-derived PDGF-BB is significantly associated with prolonged survival (log-rank test, $p=0.038$ ). (B) Overexpression of tumor-derived MMP-1 is significantly associated with prolonged survival (logrank test, $p=0.029$ ). (C) Overexpression of tumor-derived MMP-2 is an indicator for a better prognosis and shows a trend for an improved clinical outcome $(p=0.058)$.

Table 2: Univariate analysis (log-rank test) of 9 angiogenic cytokines and 5 MMPs for median cancer-specific survival. Samples were grouped as low $(\leq$ median expression of angiogenic cytokines or MMPs) or high ( $>$ median expression of angiogenic cytokines or MMPs) level of expression and were analyzed separately from pancreatic tumor cells (tumor), surrounding tumor stroma (stroma) and corresponding serum.

\begin{tabular}{|c|c|c|c|c|}
\hline \multirow{2}{*}{ Cytokines/MMPs } & \multirow[t]{2}{*}{ Compartment } & \multicolumn{2}{|c|}{ Cancer-specific Survival (Months) } & \multirow{2}{*}{$p$-Value } \\
\hline & & Low & High & \\
\hline \multirow{3}{*}{ Angiopoietin-2 } & Tumor & 18.0 & 19.0 & 0.92 \\
\hline & Stroma & 18.0 & 19.0 & 0.41 \\
\hline & Serum & 19.0 & 15.0 & 0.36 \\
\hline \multirow{3}{*}{ Follistatin } & Tumor & 23.0 & 18.0 & 0.59 \\
\hline & Stroma & 23.0 & 16.0 & 0.789 \\
\hline & Serum & 16.0 & 23.0 & 0.4 \\
\hline \multirow{3}{*}{ G-CSF } & Tumor & 18.0 & 23.0 & 0.37 \\
\hline & Stroma & 19.0 & 19.0 & 0.99 \\
\hline & Serum & 19.0 & 18.0 & 0.417 \\
\hline \multirow{3}{*}{ HGF } & Tumor & 18.0 & 23.0 & 0.46 \\
\hline & Stroma & 33.0 & 19.0 & 0.35 \\
\hline & Serum & 18.0 & 23.0 & 0.537 \\
\hline \multirow{3}{*}{ IL-8 } & Tumor & 18.0 & 23.0 & 0.59 \\
\hline & Stroma & 18.0 & 19.0 & 0.41 \\
\hline & Serum & 16.0 & 23.0 & 0.495 \\
\hline \multirow{3}{*}{ Leptin } & Tumor & 19.0 & 18.0 & 0.37 \\
\hline & Stroma & 23.0 & 16.0 & 0.26 \\
\hline & Serum & 19.0 & 16.0 & 0.301 \\
\hline
\end{tabular}

(Continued) 


\begin{tabular}{|c|c|c|c|c|}
\hline \multirow{2}{*}{ Cytokines/MMPs } & \multirow[t]{2}{*}{ Compartment } & \multicolumn{2}{|c|}{ Cancer-specific Survival (Months) } & \multirow{2}{*}{$p$-Value } \\
\hline & & Low & High & \\
\hline \multirow{3}{*}{ PDGF-BB } & Tumor & 15.0 & Median not reached & 0.038 \\
\hline & Stroma & 18.0 & 19.0 & 0.26 \\
\hline & Serum & 18.0 & 26.0 & 0.356 \\
\hline \multirow{3}{*}{ PECAM-1 } & Tumor & 16.0 & 23.0 & 0.57 \\
\hline & Stroma & 26.0 & 15.0 & 0.19 \\
\hline & Serum & 18.0 & 26.0 & 0.257 \\
\hline \multirow{3}{*}{ VEGF } & Tumor & 18.0 & 23.0 & 0.49 \\
\hline & Stroma & 16.0 & 43.0 & 0.26 \\
\hline & Serum & 19.0 & 43.0 & 0.573 \\
\hline \multirow{3}{*}{ MMP-1 } & Tumor & 15.0 & 23.0 & 0.029 \\
\hline & Stroma & 16.0 & 19.0 & 0.53 \\
\hline & Serum & 19.0 & 15.0 & 0.122 \\
\hline \multirow{3}{*}{ MMP-2 } & Tumor & 16.0 & 33.0 & 0.058 \\
\hline & Stroma & 19.0 & 18.0 & 0.83 \\
\hline & Serum & 19.0 & 15.0 & 0.136 \\
\hline \multirow{3}{*}{ MMP-7 } & Tumor & 16.0 & 23.0 & 0.32 \\
\hline & Stroma & 16.0 & 19.0 & 0.38 \\
\hline & Serum & 19.0 & 15.0 & 0.320 \\
\hline \multirow{3}{*}{ MMP-9 } & Tumor & 16.0 & 43.0 & 0.074 \\
\hline & Stroma & 19.0 & 19.0 & 0.71 \\
\hline & Serum & 23.0 & 13.0 & 0.182 \\
\hline \multirow{3}{*}{ MMP-10 } & Tumor & 23.0 & 16.0 & 0.133 \\
\hline & Stroma & 19.0 & 18.0 & 0.49 \\
\hline & Serum & 19.0 & 18.0 & 0.459 \\
\hline
\end{tabular}

Table 3: Multivariate analysis (Cox proportional hazards regression model) of prognostic parameters for cancer-specific overall survival in pancreatic cancer. (CI: confidence interval).

\begin{tabular}{|l|l|l|l|l|}
\hline Characteristics & \multicolumn{1}{l}{ Category } & Hazard ratio & $\mathbf{9 5 \%}$ CI of Relative Risk & \multicolumn{1}{l}{$\boldsymbol{P}$-Value } \\
\hline Gender & (Male/Female) & 1.728 & $0.576-5.181$ & 0.329 \\
\hline MedianAge & $\leq 70$ years vs. $>$ 70 years & 0.369 & $0.144-0.949$ & $\mathbf{0 . 0 3 9}$ \\
\hline T-stage & pT3 vs. pT4 & 0.165 & $0.017-1.579$ & 0.118 \\
\hline N-stage & pN0 vs. pN1 & 2.344 & $0.652-8.426$ & 0.192 \\
\hline M-stage & M0 vs. M1 & 1.033 & $0.197-5.415$ & 0.97 \\
\hline Tumorgrade & G2 vs. G3 & 2.787 & $0.96-8.094$ & 0.06 \\
\hline Resection status & R0 vs. R1 & 1.322 & $0.284-6.152$ & 0.722 \\
\hline Tumor PDGF & Low vs. high expression & 0.265 & $0.101-0.693$ & $\mathbf{0 . 0 0 7}$ \\
\hline Tumor MMP-1 & Low vs. high expression & 0.347 & $0.121-0.996$ & $\mathbf{0 . 0 4 9}$ \\
\hline Tumor MMP-2 & Low vs. high expression & 0.616 & $0.208-1.828$ & 0.383 \\
\hline
\end{tabular}


pancreatic stellate cells are implicated in neoangiogenesis by activating the c-MET pathway through the release of HGF [23]. In contrast, Rhim et al. have shown that hedgehog-driven stroma suppresses tumor growth in part by restraining tumor angiogenesis [24].

Our study presents a detailed expression profile of angiogenic biomarkers in pancreatic cancer cells, as well as surrounding tumor stroma and corresponding serum samples. These findings reflect the dichotomous stimulating and inhibiting roles of tumor stroma in pancreatic cancer: consistent with previous reports $[25,26]$, our data show a tendancy that tumor-adjacent stroma is the major source of several pro-angiogenic cytokines and MMPs (e.g. HGF, MMP-2, and G-CSF). Likewise, the stroma component displayed a significantly enhanced release of follistatin, which can suppress the formation of multiple organ metastasis predominantly by inhibiting angiogenesis [27]. These findings support the hypothesis that pancreatic tumor stroma is not uniform but its cellular heterogeneity can exert tumor-promoting as well as host-protective functions by impairing neovascularization. In this context, it can be conjectured that the hypoxic and hypovascular microenvironment in pancreatic cancer [28, 29] is a surrogate marker for the host defense mechanism of the host tissue against cancer cells. However, from a therapeutical point of view, a strong desmoplastic and hypovascular tumor-associated stroma is a double-edged sword: on the one hand it may constitute a protective response from the host against tumor cells and act as a physical barrier against tumor cell invasion [30]. On the other hand, the hypovascular stroma impairs the delivery of cytotoxic chemotherapies to the peritumoral milieu [30, 31]. This might be one reason that the combination of standard chemotherapies such as gemcitabine with antiangiogenic therapies such as bevacizumab have failed to improve the outcome in patients with pancreatic cancer [32].

Additionally, since circulating MMPs and angiogenic cytokines have been proposed as relevant non-invasive surrogate markers in tumor disease [33-35], we compared compartment-specific expression between serum, cancer cells and stromal cells of the same patient. An unsupervised hierachical clustering based on the expression of angiogenic cytokines and MMPs did not reveal any distinct subsets of patients for tumor-, serum-, or stroma-derived factors. Similarly, no distinct clusters were apparent when supervising cluster analysis based on patient tumor grade or UICC stage of the primary cancer. Based on these findings, one may assume that a panel of 17 angiogenic factors is not specific enough to identify molecular subgroups of pancreatic cancer which have a distinct expression profile. To determine different biological subtypes of pancreatic cancer, other tools such as RNA expression analysis or RNA sequencing might be preferred as they have a more informative value to adress this question [36-38]. Furthermore, we found the majority of angiogenic cytokines and MMPs to be more highly expressed in serum, albeit without any significant linear relationship between pancreatic cancer tissue and serum for any angiogenic cytokine or MMP. These results are consistent with previous reports investigating the correlation between tissue tumor biomarkers and corresponding serum samples [39, 40]. Our findings could be explained by the presence of immunocompetent cells in the blood, which are a known source of circulating angiogenic cytokines and MMPs [41]. In light of this, serum-derived biomarkers do not only reflect the expression profile of the solid tumor, but also represent the inflammatory host response. This qualifies circulating angiogenic factors as being useful surrogate markers for tumor diagnosis and prognosis. However, due to the heterogenous source of circulating factors, they offer limited insight into tumor biology itself. This may also explain why the unsupervised cluster analysis of angiogenic serum markers did not reveal any specific subgroups which were associated with a more aggressive tumor type or a more advanced tumor stage.

Consistent with their presumptive role as biomarkers, we have identifed MMP-1,-3,-7,-9,-10 and 12 as being upregulated in the serum of patients with pancreatic cancer, as compared to a cohort of healthy donors. Among those, MMP-7 and MMP-12 displayed high sensitivity and specificity in discriminating between serum samples of pancreatic cancer patients and healthy donors. Even in a small subgroup of patients with early stage pancreatic cancer (UICC II), MMP-7 and MMP-12 were good classifiers to distinguish between patients with pancreatic cancer and healthy donors. These findings advocate MMPs as having a high potential as diagnostic serum markers for pancreatic cancer. Moreover, they may offer a more cost-effective and simply applicable alternative with a similar diagnostic value in comparison to other fluid biomarkers such as microRNAs [42]. However, further studies are required to unravel the question of whether elevated serum MMPs are specific for pancreatic cancer, or are increased in other tumor types or chronic diseases as well.

To assess wether these proteins could predict more specific disease properties, we assessed the compartmentspecific expression profiles of angiogenic cytokines and MMPs in correlation with histopathological data and disease prognosis. Our findings indicated that while tumor-derived angiogenic cytokines and MMPs are potential prognostic biomarkers, stroma- and serumderived factors did not show any significant association between expression level and survival. Low expression of tumor-derived PDGF-BB correlated significantly with a decreased cancer-specific survial. PDGF-BB activates pericytes by the tyrosine kinase receptor PDGFR $\beta$ [43, 44], which likely serve as important gatekeepers against cancer progression and metastasis by stabilizing the tumor vasculature [45]. Previous data has shown that increasing the pericyte content of the 
pancreatic tumor microenvironment inhibits the growth of angiogenesis-dependent tumors [43] and that poor pericyte coverage increases hypoxic strain in breast cancer, which activates epithelial-to-mesenchymal transition and enhances metastasis [45]. Thus, we hypothesize that overexpression of tumor-derived PDGF-BB results in an increased activation of pericytes in the pancreactic cancerassociated vasculature, inhibiting the spread of tumor cells and improving prognosis.

Alternately, tumor-derived MMP-1 and -2 were associated with a prolonged survival, with MMP-1 being a prognostic factor in both the univarate and multivariate survival analysis. Though several MMPs have been claimed to contribute to tumor dissemination by supporting tumor cell vascular penetration, more recent experimental data indicate that some members of the MMP family can also exert tumor-suppressive functions [46-48]. For example, Wong et al. have observed that MMP1 expression was downregulated with advancing disease stage for both colon and rectal cancers [46]. Moreover, this study reported that patients with stage III colon cancer experienced shorter time to distant metastasis and decreased overall survival when lacking MMP-1 [46]. Furthermore, by performing a large meta-analysis of colorectal cancer highthroughput gene-expression studies, Wong et al. identified MMP2 as being lesser expressed in colorectal metastases, as compared to the primary tumor. The authors provided evidence that inhibition of MMP2 promotes cell invasion in vitro, an effect most dramatic in chemoresistant cells [46]. Thus, while the potential anti-tumorigenic role of MMP-1 and -2 requires further investigation, our findings might explain why many clinical trials in the last decade have failed when broad-spectrum MMP-inhibitors were applied to patients in an attempt to find an anticancer agent [49].

In conclusion, our study exhibits a new approach to detect potential biomarkers and therapeutic molecluar targets in pancreatic cancer. We show that the tumor-associated stroma is a major source of various angiogenic cytokines and MMPs. Moreover, we demonstrate that a panel of circulating serum MMPs is a useful tool in primary tumor to discriminate between healthy donors and patients with pancreatic cancer. These findings require further validation in subsequent clinical trials but might be helpful to detect pancreatic cancer at an early stage, hence improving the early prognosis of the disease. Finally, we have identified tumor-derived PDGF-BB and MMP-1 as novel prognostic markers. Further clinical trials may validate these factors as useful biomarkers to classify patients into different risk groups that might benefit from a more personalized therapy.

\section{MATERIALS AND METHODS}

\section{Patient characteristics and data collection}

The use of patient tissue samples and clinicopathological information in this study has been approved by the Medical Ethical Committee of the
University of Heidelberg. Written informed consent was obtained from each patient preoperatively and from each healthy donor prior to serum collection. Kryo-frozen tissue samples were obtained from patients with primary pancreatic adenocarcinomas who underwent tumor resection between 2007 and 2011 at the Department of General, Visceral, and Transplantation Surgery, University of Heidelberg. Clinical information included age, gender, UICC stage, grading, resection status and cancer-related survival (time from diagnosis to cancer-related death or last follw-up).

\section{Clinical specimens}

Kryo-frozen tumor samples were retrieved as previously described [50]. Briefly, samples were snapfrozen in liquid nitrogen immediately after resection and stored at $-80^{\circ} \mathrm{C}$. A $10 \mu \mathrm{m}$ reference section of each sample was cut and stained with hematoxylin and eosin (standard methods) to indicate the proportion of tumor tissue and adjacent tumor stroma. Serum samples of patients with pancreatic cancer were obtained by taking blood samples immediately before surgical incision while serum samples from healthy donors were obtained under standard sterile conditions. The blood samples were then centrifuged at $2,500 \mathrm{~g}$ for 10 minutes to extract the serum, which was then stored at $-80^{\circ} \mathrm{C}$ until further analysis.

\section{Microdissection}

\section{Tissue preparation and laser microdissection}

Tissue preparation for laser microdissection was performed as previously described [51]. A $10 \mu \mathrm{m}$ reference section of each sample was cut and stained with hematoxylin and eosin by standard methods to evaluate the proportion of tumor tissue and adjacent tumor stroma. Samples with a tumor stroma proportion $>30 \%$ were included into this study. Briefly, $20 \mu \mathrm{m}$ sections of kryo-frozen pancreatic cancer tissue were mounted on Zeiss membrane slides (Carl Zeiss microimaging, Jena, Germany), stained with cresyl violet using a LCM Staining Kit (Ambion ${ }^{\circledR} /$ Applied Biosystems, Darmstadt, Germany) and stored at $-80^{\circ} \mathrm{C}$ until further processing. A calculated area of $40 \mathrm{~mm}^{2}$ of tumor epithelial tissue and $40 \mathrm{~mm}^{2}$ of surrounding tumor stroma were obtained separately by laser microdissection (PALM Microbeam, Carl Zeiss microimaging, Jena, Germany) and stored in an adhesive cap (Carl Zeiss, Jena, Germany) immediately at $-80^{\circ} \mathrm{C}$ until further processing.

\section{Protein lysates}

Tissue samples were lysed in Bio-Plex Lysis Buffer, and protein concentrations determined using a BCA protein assay kit (Thermo Scientific, 58239 Schwerte, Germany). Lysates were adjusted to a total protein concentration of $150 \mu \mathrm{g} / \mathrm{ml}$ and $7.5 \mu \mathrm{g}$ protein $(50 \mu \mathrm{L})$. Serum samples 
from both tumor patients and controls were centrifuged and either diluted 1:20 with assay buffer (for panel 1 of the MMP-assay) or used undiluted (for the other assays) according to the manufacturer's instructions. $40 \mu \mathrm{l}$ of the diluted and undiluted serum samples were transferred into a 96-well plate and immediately analyzed. Microdissected tissue and serum samples were quantified using the Bio-Plex Human Angiogenesis Assay (Bio-Rad Laboratories, Inc., Hercules, CA 94547 USA) and the Millipore MILLIPLEX MAP Human MMP Panel 1 and 2 (Millipore 290 Concord Road, Billerica, MA) according to the manufacturer's instructions and as described recently [13]. These panels included the following proteins: angiopoietin-2, follistatin, granulocyte colony-stimulating-factor (G-CSF), hepatocyte growth factor (HGF), interleukin-8 (IL-8, CXCL8), leptin, platelet-derived growth factor beta (PDGF-BB), platelet endothelial cell adhesion molecule-1 (PECAM-1, sCD31), vascular endothelial growth factor (VEGF), and matrix metalloproteinases (MMPs)-1, -2, -3, -7, -9, -10, -12, and -13 . Standard curves and concentrations were calculated with Bio-Plex Manager 4.1.1 using a 5-parameter logistic regression formula.

\section{Statistical analysis}

The SPSS software package version 21.0 (Chicago, IL, USA) and the R statistical framework, version 2.15 (http://www.r-project.org) were used for all calculations. Pairwisse Student $t$ tests were performed to compare the expression of angiogenic factors in pancreatic cancer cells, tumor stroma and corresponding serum and Bonferroni correction was applied to allow for multiple testing. Correlations between the expression of tissue-derived and serum-derived factors were determined using Pearson's correlation. Positive correlations are reported at a Pearson's coefficient $(r)>0.5$, while inverse correlation are at $r<-0.5$. Chi squared $\left(\chi^{2}\right)$ tests were applied to examine correlation between the expression of angiogenic factors and clinical and pathological parameters and Bonferroni correction was applied to allow for multiple testing. Heatmaps were drawn using the enhanced heatmap package (http:// hosho.ees.hokudai.ac.jp/ kubo/Rdoc/library/gplots/html/heat map.2.html), and area under the receiver operating characteristic curve (AUC)/sensitivity analysis was done using the ROCR package [52], both for R. Univariate survival analysis was performed using the KaplanMeier method, with survival differences calculated with the log-rank test. Multivariable analysis (Cox proportional hazards regression model) of cancer-specific survival was performed on all covariates that showed significant association with all relevant clinicopathological parameters in the univariate analysis.

\section{ACKNOWLEDGEMENTS}

We thank Ludmila Umansky and Tina Lerchl for excellent technical assistance for BioPlex Assays.

\section{Disclosure/conflict of interest}

The authors declare no potential conflicts of interest.

\begin{abstract}
Abbreviations
G-CSF, granulocyte colony-stimulating-factor; HGF, hepatocyte growth factor; IL-8, interleukin-8; PDGF-BB, platelet-derived growth factor beta; PECAM-1, platelet endothelial cell adhesion molecule-1; VEGF, vascular endothelial growth factor; MMP, matrix metalloproteinase.
\end{abstract}

\section{REFERENCES}

1. Siegel R, Naishadham D, Jemal A. Cancer statistics. CA Cancer J Clin. 2013; 63:11-30.

2. Hariharan D, Saied A, Kocher HM. Analysis of mortality rates for pancreatic cancer across the world. HPB (Oxford). 2008; 10:58-62.

3. Werner J, Combs SE, Springfeld C, Hartwig W, Hackert T, Buchler MW. Advanced-stage pancreatic cancer: therapy options. Nature reviews Clinical oncology. 2013; 10:323-333.

4. Conroy T, Desseigne F, Ychou M, Bouche O, Guimbaud R, Becouarn Y, Adenis A, Raoul JL, Gourgou-Bourgade S, de la Fouchardiere C, Bennouna J, Bachet JB, Khemissa-Akouz F, Pere-Verge D, Delbaldo C, Assenat E, et al. FOLFIRINOX versus gemcitabine for metastatic pancreatic cancer. The New England journal of medicine. 2011; 364:1817-1825.

5. Von Hoff DD, Ervin T, Arena FP, Chiorean EG, Infante J, Moore M, Seay T, Tjulandin SA, Ma WW, Saleh MN, Harris M, Reni M, Dowden S, Laheru D, Bahary N, Ramanathan RK, et al. Increased survival in pancreatic cancer with nab-paclitaxel plus gemcitabine. The New England journal of medicine. 2013; 369:1691-1703.

6. Schmidt J, Abel U, Debus J, Harig S, Hoffmann K, Herrmann T, Bartsch D, Klein J, Mansmann U, Jager D, Capussotti L, Kunz R, Buchler MW. Open-label, multicenter, randomized phase III trial of adjuvant chemoradiation plus interferon Alfa- $2 b$ versus fluorouracil and folinic acid for patients with resected pancreatic adenocarcinoma. J Clin Oncol. 2012; 30:4077-4083.

7. Costello E, Greenhalf W, Neoptolemos JP. New biomarkers and targets in pancreatic cancer and their application to treatment. Nat Rev Gastroenterol Hepatol. 2012; 9:435-444.

8. Delcore R, Rodriguez FJ, Forster J, Hermreck AS, Thomas JH. Significance of lymph node metastases in patients with pancreatic cancer undergoing curative resection. Am J Surg. 1996; 172:463-468; discussion 468-469.

9. Geer RJ, Brennan MF. Prognostic indicators for survival after resection of pancreatic adenocarcinoma. Am J Surg. 1993; 165:68-72; discussion 72-63. 
10. Hellan M, Sun CL, Artinyan A, Mojica-Manosa P, Bhatia S, Ellenhorn JD, Kim J. The impact of lymph node number on survival in patients with lymph node-negative pancreatic cancer. Pancreas. 2008; 37:19-24.

11. Sund M, Kalluri R. Tumor stroma derived biomarkers in cancer. Cancer Metastasis Rev. 2009; 28:177-183.

12. Rahbari NN, Schmidt T, Falk CS, Hinz U, Herber M, Bork U, Buchler MW, Weitz J, Koch M. Expression and prognostic value of circulating angiogenic cytokines in pancreatic cancer. BMC Cancer. 2011; 11:286.

13. Halama N, Braun M, Kahlert C, Spille A, Quack C, Rahbari N, Koch M, Weitz J, Kloor M, Zoernig I, Schirmacher P, Brand K, Grabe N, Falk CS. Natural killer cells are scarce in colorectal carcinoma tissue despite high levels of chemokines and cytokines. Clin Cancer Res. 2011; 17:678-689.

14. Szkandera J, Stotz M, Absenger G, Stojakovic T, Samonigg H, Kornprat P, Schaberl-Moser R, Alzoughbi W, Lackner C, Ress AL, Seggewies FS, Gerger A, Hoefler G, Pichler M. Validation of C-reactive protein levels as a prognostic indicator for survival in a large cohort of pancreatic cancer patients. British journal of cancer. 2014; 110:183-188.

15. Stotz M, Gerger A, Eisner F, Szkandera J, Loibner H, Ress AL, Kornprat P, AlZoughbi W, Seggewies FS, Lackner C, Stojakovic T, Samonigg H, Hoefler G, Pichler M. Increased neutrophil-lymphocyte ratio is a poor prognostic factor in patients with primary operable and inoperable pancreatic cancer. British journal of cancer. 2013; 109:416-421.

16. Erkan M, Hausmann S, Michalski CW, Fingerle AA, Dobritz M, Kleeff J, Friess H. The role of stroma in pancreatic cancer: diagnostic and therapeutic implications. Nat Rev Gastroenterol Hepatol. 2012; 9:454-467.

17. Feig C, Gopinathan A, Neesse A, Chan DS, Cook N, Tuveson DA. The pancreas cancer microenvironment. Clinical cancer research: an official journal of the American Association for Cancer Research. 2012; 18:4266-4276.

18. Kessenbrock K, Plaks V, Werb Z. Matrix metalloproteinases: regulators of the tumor microenvironment. Cell. 2010; 141: 52-67.

19. Vong S, Kalluri R. The role of stromal myofibroblast and extracellular matrix in tumor angiogenesis. Genes Cancer. 2011; 2:1139-1145.

20. Neesse A, Michl P, Frese KK, Feig C, Cook N, Jacobetz MA, Lolkema MP, Buchholz M, Olive KP, Gress TM, Tuveson DA. Stromal biology and therapy in pancreatic cancer. Gut. 2011; 60:861-868.

21. Verbeke CS, Leitch D, Menon KV, McMahon MJ, Guillou PJ, Anthoney A. Redefining the R1 resection in pancreatic cancer. The British journal of surgery. 2006; 93:1232-1237.

22. Sakamoto H, Kimura H, Sekijima M, Matsumoto K, Arao T, Chikugo T, Yamada Y, Kitano M, Ito A,
Takeyama Y, Kudo M, Nishio K. Plasma concentrations of angiogenesis-related molecules in patients with pancreatic cancer. Japanese journal of clinical oncology. 2012; 42:105-112.

23. Patel MB, Pothula SP, Xu Z, Lee AK, Goldstein D, Pirola RC, Apte MV, Wilson JS. The role of the hepatocyte growth factor/c-MET pathway in pancreatic stellate cellendothelial cell interactions: antiangiogenic implications in pancreatic cancer. Carcinogenesis. 2014; 35:1891-1900.

24. Rhim AD, Oberstein PE, Thomas DH, Mirek ET, Palermo CF, Sastra SA, Dekleva EN, Saunders T, Becerra CP, Tattersall IW, Westphalen CB, Kitajewski J, Fernandez-Barrena MG, Fernandez-Zapico ME, Iacobuzio-Donahue $\mathrm{C}$, Olive KP, et al. Stromal elements act to restrain, rather than support, pancreatic ductal adenocarcinoma. Cancer cell. 2014; 25:735-747.

25. Gong YL, Xu GM, Huang WD, Chen LB. Expression of matrix metalloproteinases and the tissue inhibitors of metalloproteinases and their local invasiveness and metastasis in Chinese human pancreatic cancer. J Surg Oncol. 2000; 73:95-99.

26. Ide T, Kitajima Y, Miyoshi A, Ohtsuka T, Mitsuno M, Ohtaka K, Miyazaki K. The hypoxic environment in tumorstromal cells accelerates pancreatic cancer progression via the activation of paracrine hepatocyte growth factor/c-Met signaling. Ann Surg Oncol. 2007; 14:2600-2607.

27. Ogino H, Yano S, Kakiuchi S, Muguruma H, Ikuta K, Hanibuchi M, Uehara H, Tsuchida K, Sugino H, Sone S. Follistatin suppresses the production of experimental multiple-organ metastasis by small cell lung cancer cells in natural killer cell-depleted SCID mice. Clinical cancer research: an official journal of the American Association for Cancer Research. 2008; 14:660-667.

28. Komar G, Kauhanen S, Liukko K, Seppanen M, Kajander S, Ovaska J, Nuutila P, Minn H. Decreased blood flow with increased metabolic activity: a novel sign of pancreatic tumor aggressiveness. Clinical cancer research: an official journal of the American Association for Cancer Research. 2009; 15:5511-5517.

29. Olson P, Chu GC, Perry SR, Nolan-Stevaux O, Hanahan D. Imaging guided trials of the angiogenesis inhibitor sunitinib in mouse models predict efficacy in pancreatic neuroendocrine but not ductal carcinoma. Proceedings of the National Academy of Sciences of the United States of America. 2011; 108:E1275-1284.

30. Ozdemir BC, Pentcheva-Hoang T, Carstens JL, Zheng X, Wu CC, Simpson TR, Laklai H, Sugimoto H, Kahlert C, Novitskiy SV, De Jesus-Acosta A, Sharma P, Heidari P, Mahmood U, Chin L, Moses HL, et al. Depletion of carcinoma-associated fibroblasts and fibrosis induces immunosuppression and accelerates pancreas cancer with reduced survival. Cancer cell. 2014; 25:719-734.

31. Provenzano PP, Cuevas C, Chang AE, Goel VK, Von Hoff DD, Hingorani SR. Enzymatic targeting of the 
stroma ablates physical barriers to treatment of pancreatic ductal adenocarcinoma. Cancer cell. 2012; 21:418-429.

32. Van Cutsem E, Vervenne WL, Bennouna J, Humblet Y, Gill S, Van Laethem JL, Verslype C, Scheithauer W, Shang A, Cosaert J, Moore MJ. Phase III trial of bevacizumab in combination with gemcitabine and erlotinib in patients with metastatic pancreatic cancer. Journal of clinical oncology: official journal of the American Society of Clinical Oncology. 2009; 27:2231-2237.

33. Park HD, Kang ES, Kim JW, Lee KT, Lee KH, Park YS, Park JO, Lee J, Heo JS, Choi SH, Choi DW, Kim S, Lee JK, Lee SY. Serum CA19-9, cathepsin D, and matrix metalloproteinase-7 as a diagnostic panel for pancreatic ductal adenocarcinoma. Proteomics. 2012; 12:3590-3597.

34. Wilson S, Damery S, Stocken DD, Dowswell G, Holder R, Ward ST, Redman V, Wakelam MJ, James J, Hobbs FD, Ismail T. Serum matrix metalloproteinase 9 and colorectal neoplasia: a community-based evaluation of a potential diagnostic test. British journal of cancer. 2012; 106:1431-1438.

35. Rahbari NN, Reissfelder C, Muhlbayer M, Weidmann K, Kahlert C, Buchler MW, Weitz J, Koch M. Correlation of circulating angiogenic factors with circulating tumor cells and disease recurrence in patients undergoing curative resection for colorectal liver metastases. Annals of surgical oncology. 2011; 18:2182-2191.

36. Vilardell F, Iacobuzio-Donahue CA. Cancer gene profiling in pancreatic cancer. Methods in molecular biology. 2010; 576:279-292.

37. Pilarsky C, Grutzmann R. Genomics of pancreatic ductal adenocarcinoma. Hepatobiliary \& pancreatic diseases international: HBPD INT. 2014; 13:381-385.

38. Grutzmann R, Boriss H, Ammerpohl O, Luttges J, Kalthoff H, Schackert HK, Kloppel G, Saeger HD, Pilarsky C. Meta-analysis of microarray data on pancreatic cancer defines a set of commonly dysregulated genes. Oncogene. 2005; 24:5079-5088.

39. Tachezy M, Zander H, Marx AH, Stahl PR, Gebauer F, Izbicki JR, Bockhorn M. ALCAM (CD166) expression and serum levels in pancreatic cancer. PloS one. 2012; 7:e39018.

40. Tachezy M, Effenberger $\mathrm{K}$, Zander H, Minner S, Gebauer F, Vashist YK, Sauter G, Pantel K, Izbicki JR, Bockhorn M. ALCAM (CD166) expression and serum levels are markers for poor survival of esophageal cancer patients. International journal of cancer Journal international du cancer. 2012; 131:396-405.

41. Smith HA, Kang Y. The metastasis-promoting roles of tumor-associated immune cells. J Mol Med (Berl). 2013.

42. Schultz NA, Dehlendorff C, Jensen BV, Bjerregaard JK, Nielsen KR, Bojesen SE, Calatayud D, Nielsen SE,
Yilmaz M, Hollander NH, Andersen KK, Johansen JS. MicroRNA biomarkers in whole blood for detection of pancreatic cancer. Jama. 2014; 311:392-404.

43. McCarty MF, Somcio RJ, Stoeltzing O, Wey J, Fan F, Liu W, Bucana C, Ellis LM. Overexpression of PDGF-BB decreases colorectal and pancreatic cancer growth by increasing tumor pericyte content. The Journal of clinical investigation. 2007; 117:2114-2122.

44. LeBleu VS, Kalluri R. Blockade of PDGF receptor signaling reduces myofibroblast number and attenuates renal fibrosis. Kidney international. 2011; 80:1119-1121.

45. Cooke VG, LeBleu VS, Keskin D, Khan Z, O’Connell JT, Teng Y, Duncan MB, Xie L, Maeda G, Vong S, Sugimoto H, Rocha RM, Damascena A, Brentani RR, Kalluri R. Pericyte depletion results in hypoxia-associated epithelialto-mesenchymal transition and metastasis mediated by met signaling pathway. Cancer cell. 2012; 21:66-81.

46. Wong JC, Chan SK, Schaeffer DF, Sagaert X, Lim HJ, Kennecke H, Owen DA, Suh KW, Kim YB, Tai IT. Absence of MMP2 expression correlates with poor clinical outcomes in rectal cancer, and is distinct from MMP1related outcomes in colon cancer. Clinical cancer research: an official journal of the American Association for Cancer Research. 2011; 17:4167-4176.

47. Agesen TH, Sveen A, Merok MA, Lind GE, Nesbakken A, Skotheim RI, Lothe RA. ColoGuideEx: a robust gene classifier specific for stage II colorectal cancer prognosis. Gut. 2012; 61:1560-1567.

48. Decock J, Thirkettle S, Wagstaff L, Edwards DR. Matrix metalloproteinases: protective roles in cancer. Journal of cellular and molecular medicine. 2011; 15:1254-1265.

49. Coussens LM, Fingleton B, Matrisian LM. Matrix metalloproteinase inhibitors and cancer: trials and tribulations. Science. 2002; 295:2387-2392.

50. Kahlert C, Klupp F, Brand K, Lasitschka F, Diederichs S, Kirchberg J, Rahbari N, Dutta S, Bork U, Fritzmann J, Reissfelder C, Koch M, Weitz J. Invasion front-specific expression and prognostic significance of microRNA in colorectal liver metastases. Cancer Sci. 2011; 102:1799-1807.

51. Kahlert C, Lahes S, Radhakrishnan P, Dutta S, Mogler C, Herpel E, Brand K, Steinert G, Schneider M, Mollenhauer M, Reissfelder C, Klupp F, Fritzmann J, Wunder C, Benner A, Kloor M, et al. Overexpression of ZEB2 at the invasion front of colorectal cancer is an independent prognostic marker and regulates tumor invasion in vitro. Clinical cancer research: an official journal of the American Association for Cancer Research. 2011; 17:7654-7663.

52. Sing $T$, Sander O, Beerenwinkel N, Lengauer $T$. ROCR: visualizing classifier performance in $R$. Bioinformatics. 2005; 21:3940-3941. 